\section{The Chemistry of Star-Forming Regions}

\author{
DAVID A. WILLIAMS*,+ AND \\ THOMAS W. HARTQUIST‡
}

Department of Physics and Astronomy, University College London, U.K., and Department of Physics, University of Leeds, U.K.

Received J anuary 26, 1998

\section{Introduction}

During the last quarter century, chemists have responded magnificently to the challenges raised by astronomers in their attempts to understand the variety of molecules detected in interstellar clouds. Observations have shown the chemistry of these regions to be surprisingly complex, and now more than 100 molecular species have been identified in interstellar and circumstellar regions of the Galaxy. The chemistry of interstellar clouds that gives rise to these molecules is now believed to be reasonably well understood (see section 2) in terms of a network of some thousands of binary reactions between several hundred species. $^{1,2}$

Astronomers are now applying the techniques of astrochemistry to interpret observations of star-forming regions. ${ }^{3}$ These regions are much more complex in physical terms than quiescent interstellar clouds. In starforming regions, interstellar gas is being compressed, the force of gravity overcoming the resistance provided by gas pressure, magnetohydro-dynamic (MHD) turbulence, magnetic pressure, and rotation. The chemistry is not in steady state during this collapse, and can therefore be used as a tracer of the evolution of the collapse. In addition, the chemistry modifies and controls the collapse through the provision of molecular coolants of the gas, and by determining the fractional ionization in the gas. It is this ionization that affects the level of magnetic and turbulent support available to the cloud. Molecular rotational emissions at millimeter and submillimeter wavelengths are both the main cooling processes and the most effective probes of these regions.

David A. Williams was born in 1937 in Nottingham, U.K., educated at Queen's University Belfast (B.SC., Ph.D.); he obtained a D.Sc. from the University of Manchester in 1980. He established a molecular astrophysics group at UM IST, Manchester, formerly was a professor at UM IST 1984-1994 and currently is Perren Professor of Astronomy at University College London. Current interests include cosmic dust.

Thomas W. Harquist was born 1954 in Redwood City, CA, and was eduated Rice University (B.A.) and Harvard University (M.A., Ph.D.). He was a postdoc at Harvard, a Royal Society J affe Donation Fellow in University College London, assistant professor University of Maryland, senior research scientist M PI fuer extraterrestrische Physik, and lecturer University of Leeds, U.K. His main interest is diffuse astrophysical media.
Interstellar gas in the Galaxy is observed to be distributed in an irregular fashion, in clouds of a range of sizes. Much of the mass is encompassed in so-called giant molecular clouds (GMCs) which range in mass from about $10^{4}$ to about $10^{6}$ solar masses (the solar mass is about 2 $\times 10^{30} \mathrm{~kg}$ ), and have linear extents of several hundred light years (a light year, ly, is about $1 \times 10^{16} \mathrm{~m}$ ). The gas in GMCs is largely $\mathrm{H}_{2}$, but because that molecule has no dipole moment, the material is most effectively traced in the 1-0 rotational emission of $\mathrm{CO}$, the next most abundant molecule ( $\mathrm{CO} / \mathrm{H}_{2} \simeq 10^{-4}$ by number). Isotopomers of $\mathrm{CO}$ are also used. The $\mathrm{CO}$ emission identifies cold gas of number density $\sim 10^{3} \mathrm{H}_{2}$ molecules $\mathrm{cm}^{-3}$.

A detailed study ${ }^{4}$ of one particular GMC, the Rosette molecular cloud (RMC), shows that it contains almost 2 $\times 10^{5}$ solar masses of gas, extending over $100 \mathrm{ly}$. The gas in the RMC is fragmented into about 70 clumps with masses ranging from a few tens to a few thousands of solar masses. The clumps are embedded in a more tenuous medium, typically contain $10^{2}-10^{3} \mathrm{H}_{2}$ molecules $\mathrm{cm}^{-3}$, and are cool $(\leqslant 30 \mathrm{~K})$. Observations show that clumps with larger column densities of $\mathrm{CO}\left(\approx 10^{16} \mathrm{CO}\right.$ molecules $\left.\mathrm{cm}^{-2}\right)$ are more likely to contain embedded stars. Therefore, clumps satisfying this criterion are likely to be the sites of star formation in the RMC.

Collapse of a clump leads to fragmentation and the formation of a cluster of dense cores. Carbon monoxide $\left({ }^{12} \mathrm{C}^{16} \mathrm{O}\right)$ is not an effective tracer of gas in dense cores because the $\mathrm{CO}$ lines are optically thick and CO level populations are thermalized at lower densities. However, species of lower abundance than ${ }^{12} \mathrm{C}^{16} \mathrm{O}$ can trace the dense gas in cores $\left(\approx 10^{4} \mathrm{H}_{2}\right.$ molecules $\left.\mathrm{cm}^{-3}\right)$, and they include $\mathrm{NH}_{3}, \mathrm{CN}, \mathrm{H}_{2} \mathrm{CO}$, and CS. A typical core cluster 5 is illustrated in Figure 1. It is a contour map in intensity of 1-0 rotational emission from the minor isotopic species ${ }^{12} \mathrm{C}^{18} \mathrm{O}$. This core cluster contains cores which may evolve to form new stars. Several stars have al ready formed and are detected as infrared sources (IRS 1-4).

A primary goal of astrophysics is the detailed study of the collapse of a dense core to form a star. It is also important to gain an understanding of how gravity overcomes the various resistances to collapse, and how a young star interacts with its environment through the stellar winds and jets that develop at the earliest stages of the star's existence. The answers to these questions are certainly contained in the emissions from the molecules and dust present in the collapsing core. Identifying a collapsing core is observationally difficult. The indicators should be molecular lines that are broadened by the infalling velocities. In this Account, we describe how the search for the infall signature has led to a recognition that the interaction of gas and dust in the infalling gas produces profound changes to the chemistry and physics of star-forming regions. This interaction is poorly understood, and the nature of the star-forming process will

\footnotetext{
+ University College London.

₹ University of Leeds.
}

10.1021/ar9701140 CCC: $\$ 18.00$

() 1999 American Chemical Society Published on Web 01/20/1999 
remain obscure until these microscopic interactions can be accurately described.

There was in the 1970s and 1980s a concerted effort by experimental and theoretical chemists to solve problems identified by astronomers of gas phase chemistry in interstellar clouds. A similar program for gas/surface interactions is now required for the resolution of the larger and more significant problem of star formation.

\section{The Chemistry of Star Formation}

2.1. Basic Chemical Schemes. The parent of the star was the core; its grand parent was the clump. The onset of the chemistry at the start of this process is due to the formation of $\mathrm{H}_{2}$, believed to result from the interaction of atomic hydrogen gas with dust grains in heterogeneous catalysis. The process is not well understood, partly because of uncertainty associated with the nature of the dust surfaces. We return to the discussion of $\mathrm{H}_{2}$ formation in section 4. The nature and properties of cosmic dust were discussed by Whittet, ${ }^{6}$ and by Mathis. ${ }^{7}$ The main effects of dust that need concern us here are these: dust extinguishes starlight and provides a surface on which molecules may freeze out, and on which chemistry may occur.

We first consider the chemistry of regions sufficiently darkened by the dust in them that photons of external origin have a negligible effect on the chemistry. The interstellar medium is swept by cosmic rays, which are mainly fast protons and electrons with energies of several hundred $\mathrm{MeV}$. Cosmic rays ( $\mathrm{cr}$ ) cause ionization of $\mathrm{H}_{2}$ and He:

$$
\begin{gathered}
\mathrm{H}_{2} \stackrel{\mathrm{cr}}{\longrightarrow} \mathrm{H}_{2} \stackrel{+}{\stackrel{\mathrm{H}_{2}}{\longrightarrow}} \mathrm{H}_{3}^{+} \\
\mathrm{He} \stackrel{\mathrm{cr}}{\longrightarrow} \mathrm{He}^{+}
\end{gathered}
$$

$\mathrm{H}_{2}+$ is quickly converted to $\mathrm{H}_{3}{ }^{+}$. This ion and $\mathrm{He}^{+}$are largely responsible for driving an efficient ion-molecule chemistry. Because $\mathrm{H}_{2}$ is by far the most abundant species, if another species can react with it, that species will be removed primarly by $\mathrm{H}_{2}$. Thus, proton transfer followed by a sequence of hydrogen abstraction reactions is important for the chemistry of several abundant elements. For instance,

$$
\begin{aligned}
& \mathrm{C} \stackrel{\mathrm{H}_{3}^{+}}{\longrightarrow} \mathrm{CH}^{+} \stackrel{\mathrm{H}_{2}}{\longrightarrow} \mathrm{CH}_{2} \stackrel{+}{\stackrel{\mathrm{H}_{2}}{\longrightarrow}} \mathrm{CH}_{3} \stackrel{+}{\rightarrow} \mathrm{CH}, \mathrm{CH}_{2} \\
& \mathrm{CH} \stackrel{\circ}{\rightarrow} \mathrm{HCO}^{+} \stackrel{\mathrm{e}}{\rightarrow} \mathrm{CO} \stackrel{\mathrm{He}^{+}}{\longrightarrow} \mathrm{C}^{+}
\end{aligned}
$$

As illustrated above, a molecular ion that does not react nonradiatively with $\mathrm{H}_{2}$ is removed primarily by dissociative recombination (or by reactions with trace neutral species). $\mathrm{OH}$ and $\mathrm{H}_{2} \mathrm{O}$ as well as $\mathrm{NH}, \mathrm{NH}_{2}$, and $\mathrm{NH}_{3}$ are formed in a manner similar to $\mathrm{CH}$ and $\mathrm{CH}_{2}$. $\mathrm{He}^{+}$is particularly important for preventing all material from being contained in very stable species, like $\mathrm{CO}$ or $\mathrm{N}_{2}$ and $\mathrm{O}_{2}$, which are formed by the neutral-neutral reactions $\mathrm{N}$ $+\mathrm{NH} \rightarrow \mathrm{N}_{2}+\mathrm{H}$ and $\mathrm{O}+\mathrm{OH} \rightarrow \mathrm{O}_{2}+\mathrm{H}$. While $\mathrm{N}^{+}$and $\mathrm{O}^{+}$, formed by the removal of $\mathrm{N}_{2}$ and $\mathrm{O}_{2}$ in reacting with

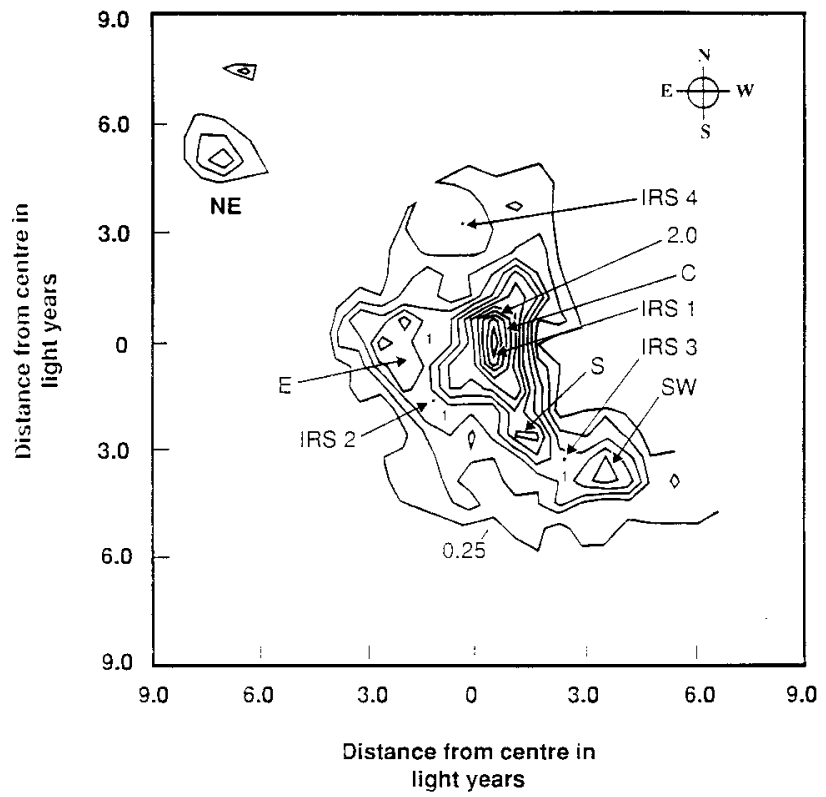

FIGURE 1. A contour map in the intensity of emission in the ${ }^{12} \mathrm{C}^{18} \mathrm{O}$ line from the molecular cloud Barnard 5. This cloud is a core-cluster, and five cores (NE, E, C, S, and SW) are evident. The cloud also contains several infrared sources (IRS 1-4); these are young stars still embedded in the dense gas from which they were formed. Reprinted with permission from ref 5. Copyright 1986 University of Chicago Press journals.

$\mathrm{He}^{+}$, react with $\mathrm{H}_{2}$, the fate of $\mathrm{C}^{+}$is more interesting and will be discussed in section 2.3.

Straightforward gas phase routes produce many of the smaller detected interstellar species. However, it appears that some contribution from surface reactions is also required. For example, the detection of interstellar $\mathrm{NH}$ in diffuse clouds 8,9 appears to need a contribution from an efficient hydrogenation of $\mathrm{N}$-atoms in surface reactions. ${ }^{10}$ Other evidence of surface chemistry arises from the study of hot cores. These are small ( $\sim 0.1 \mathrm{ly})$, dense $\left(\sim 10^{7} \mathrm{H}_{2}\right.$ $\mathrm{cm}^{-3}$ ) cores found within $\sim 1$ ly of hot stars. The intense stellar radiation heats the cores to about $100 \mathrm{~K}$, much hotter than the typical $10 \mathrm{~K}$ for interstellar matter. It is found that hot cores contain anomalously high abundances of various species (compared to quiescent molecular clouds), including some organic molecules such as ethanol, methyl formate, and dimethyl ether. Their abundances cannot be accounted for by a gas phase chemistry of the type described above. It is believed that they are formed by chemical processing of molecular ices (mostly $\mathrm{H}_{2} \mathrm{O}, \mathrm{CO}, \mathrm{CO}_{2}, \mathrm{H}_{2} \mathrm{CO}$, and $\mathrm{CH}_{3} \mathrm{OH}$ ) deposited on dust grains in the earlier, colder, prestellar phase of evolution of the material. ${ }^{11}$

2.2. Chemical Control in Star Formation. ${ }^{12}$ The collapse of a cloud converts gravitational potential energy to kinetic, much of which would appear as heat unless it was radiated away. At temperatures less than about 300 $\mathrm{K}$, the main coolant is $\mathrm{CO}$ which contains most of the carbon. The $\mathrm{CO}$ molecules radiate readily in the millimeter and submillimeter wavelength ranges. Relevant time scales, including that for cooling, are given in Table 1.

In addition to determining the cooling, the chemistry also determines the fractional ionization in the cloud. At 
Table 1. Approximate Time Scales in Star-Forming Regions

\begin{tabular}{|c|c|c|}
\hline processes & mechanism & $\begin{array}{l}\text { approximate time } \\
\text { scale (years) }\end{array}$ \\
\hline collapse & gravity & $10^{8} / \mathrm{n}_{\mathrm{H}^{1 / 2}}$ \\
\hline cooling & radiation & $\begin{array}{r}3 \times 10^{5}\left(10^{4} / \mathrm{n}_{\mathrm{H}}\right) \\
(\text { at T } \simeq 10 \mathrm{~K})\end{array}$ \\
\hline freeze-out & $\begin{array}{l}\text { gas/grain collisions, } \\
\text { sticking probability S }\end{array}$ & $3 \times 10^{9} /\left(\mathrm{n}_{\mathrm{H}} \mathrm{S}\right)$ \\
\hline $\begin{array}{l}\text { ion-molecule } \\
\text { chemistry }\end{array}$ & cosmic ray ionization & $3 \times 10^{5}$ \\
\hline $\begin{array}{l}\text { ambipolar } \\
\text { diffusion }\end{array}$ & ion-neutral drift & $4 \times 10^{5} \times(i) / 10^{-8}$ \\
\hline desorption & $\begin{array}{l}\text { chemically driven by } \\
\mathrm{H}_{2} \text { formation }\end{array}$ & $\begin{array}{c}3 \times 10^{5} \cdot\left[\mathrm{X}(\mathrm{CO}) / 10^{-4}\right] \cdot \\
{\left[1 \mathrm{~cm}^{-3} / \mathrm{n}(\mathrm{H})\right]}\end{array}$ \\
\hline
\end{tabular}

cloud edges, the carbon is almost fully photoionized and provides most of the cloud's ionization, but in clumps and cores much of the carbon is converted to $\mathrm{CO}$, which unlike most species, is screened by itself and by $\mathrm{H}_{2}$ from radiation of external origin that would otherwise dissociate it. In these conditions, the fractional ionization is a function of the number density of hydrogen nuclei $\left(n_{H}\right)$, elemental fractional abundances, and the optical depth to the cloud edge. The optical depth at visual wavelengths is caused by dust grains. Typically, one optical depth of extinction at $555 \mathrm{~nm}$ arises in a column of $2 \times 10^{21}$ hydrogen nuclei $\mathrm{cm}^{-2}$, although the grains are several times more obscuring in the far ultraviolet. For $\mathrm{n}_{\mathrm{H}} \simeq 10^{3} \mathrm{~cm}^{-3}$ and a centerto-edge column density of $2.5 \times 10^{21} \mathrm{H}_{2} \mathrm{~cm}^{-3}$ (just less than is typical of the Rosette clumps in which stars exist) $\mathrm{C}^{+}$is the dominant ion. It arises by the ionization of $\mathrm{C}$ by photons of external origin, the $C$ is released in the photodissociation of $\mathrm{CO}$ by photons radiated by the interaction of $\mathrm{H}_{2}$ with fast electrons produced when cosmic rays induce ionization. At such column densities, the fractional ionization is a rapidly decreasing function of column density. Therefore, thicker clumps have much higher damping rates of waves ${ }^{13}$ which support clumps against collapse along the large-scale magnetic field. Therefore, thicker clumps collapse sooner, giving rise to the formation of cores and ultimately stars.

At typical core densities $\mathrm{C}^{+}$is the dominant ion only in a negligibly thin outer sheath. For column densities of less than about $5 \times 10^{21} \mathrm{H}_{2} \mathrm{~cm}^{-2}$ to the edge, the dominant ions are $\mathrm{S}^{+}, \mathrm{Si}^{+}, \mathrm{Na}^{+}, \mathrm{Mg}^{+}, \mathrm{Ca}^{+}$, and other elements with low ionization potentials. For higher column densities ionization is induced primarily by cosmic rays. This leads to the formation of $\mathrm{HCO}^{+}$and other less abundant molecular ions. Charge transfer from these ions to metals such as $\mathrm{Na}$ and $\mathrm{Mg}$ produces the most abundant ions, $\mathrm{Na}^{+}$and $\mathrm{Mg}^{+}$. These atomic ions are ultimately removed in collision with dust grains carrying negative charges. In dark regions of cores, the fractional ionization is typically about $10^{-8}$. Throughout most of a core the fractional ionization depends on the fractional elemental abundances of sulfur and metals such as $\mathrm{Na}$ and $\mathrm{Mg}$. Therefore, the degree to which friction between neutrals and ions prevents neutrals from drifting during the collapse relative to magnetic field lines depends on those fractional elemental abundances. The abundances in turn

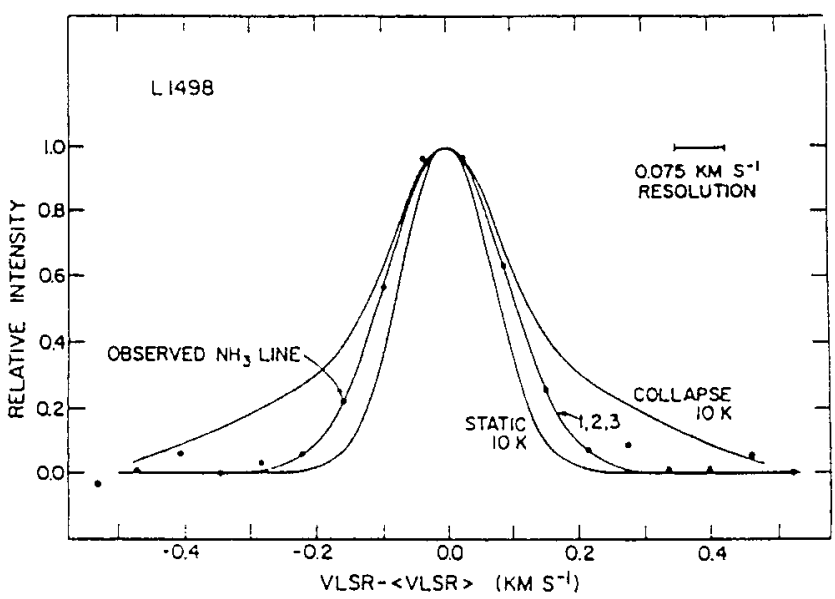

FIGURE 2. The $\mathrm{NH}_{3}$ line profile of $\mathrm{L} 1498$. The curve associated with the dots is the observed profile. The curve marked "static" is the one expected from $10 \mathrm{~K}$ gas that is not turbulent and experiences no systematic motion. The curve marked collapse is the profile expected if the fractional abundance of $\mathrm{NH}_{3}$ is constant and the core is undergoing collapse governed by a particular solution of the class that Shu (1977) investigated. Reprinted with permission from ref 14. Copyright 1983 University of Chicago Press J ournals.

are affected by freeze-out onto grains. The measurements of the abundances of gas tracers such as carbon monosulfide, CS, various molecular ions, and other gas phase species indicate that the gas phase fractional abundances of elemental sulfur and metals may be reduced by a couple of orders of magnitude, even where carbon and oxygen appear to be rather undepleted. Theoretical models of core collapse to form stars must eventually include accurate descriptions of how elemental sulfur and metals freeze onto grains.

2.3. The Detection of Core Collapse. When observational studies of dense cores began, it was expected that the signatures of collapse would be readily detected in the line profiles of molecular emissions. In the simplest picture of a collapsing core, the densest and most rapidly infalling gas would contribute broad wings to the emission profile of the radiating molecules.

However, observations of many cores did not reveal that broad profile. A detailed study in $\mathrm{NH}_{3}$ emission of one particular core that was expected to show infall gave a molecular line profile ${ }^{14}$ that deviated little from a thermal profile at $10 \mathrm{~K}$ (see Figure 2). It was suggested that perhaps the ammonia molecules expected to trace the collapse had been removed from the gas phase by freezing onto dust grains present in the gas. The existence of spectral features of dirty ices toward sufficiently opaque cores proves that such freeze-out occurs. The freeze-out rate increases with density, so the molecules in the densest parts of the core would be expected to freeze out first. A detailed study showed not only that this was a plausible explanation of the narrow $\mathrm{NH}_{3}$ line profile, but also that some other molecules behaved in a different and nonintuitive manner. While freeze-out was depleting the gas phase abundances of some molecules such as $\mathrm{H}_{2} \mathrm{O}$ and $\mathrm{NH}_{3}$, other species were actually rising in abundance. The reason for this unexpected behavior lies in the strongly coupled nature of the dense core chemistry. The $\mathrm{C}^{+}$ions 
created by reactions of $\mathrm{CO}$ with $\mathrm{He}^{+}$(see reaction (3)) can react in two main ways:

$$
\begin{gathered}
\mathrm{CO} \stackrel{\mathrm{He}^{+}}{\longrightarrow} \mathrm{C}^{+} \stackrel{\mathrm{H}_{2}}{\longrightarrow} \mathrm{CH}_{2}^{+} \rightarrow \text { hydrocarbons } \\
\mathrm{C}^{+} \stackrel{\mathrm{H}_{2} \mathrm{O}}{\longrightarrow} \mathrm{HCO}^{+} \rightarrow \mathrm{CO}
\end{gathered}
$$

The reaction of $\mathrm{C}^{+}$with $\mathrm{H}_{2}$ is a slow radiative association, whereas that with $\mathrm{H}_{2} \mathrm{O}$ is a fast ion-molecule reaction. When $\mathrm{H}_{2} \mathrm{O}$ is abundant in the gas phase, the second branch dominates and, in effect, any $\mathrm{CO}$ removed is quickly replaced. However, when $\mathrm{H}_{2} \mathrm{O}$ is depleted from the gas phase by freeze-out onto dust, carbon is effectively taken out of $\mathrm{CO}$ and put into hydrocarbons. Also, a decrease of the $\mathrm{O}$ fractional abundance reduces the $\mathrm{CH}_{3}{ }^{+}$ removal rate, increasing the fraction of $\mathrm{CH}_{3}{ }^{+}$that may react with more complicated species. As seen in Figure 3, the abundance of $\mathrm{HC}_{3} \mathrm{~N}$ responds markedly as freeze-out occurs. ${ }^{15}$ The first peak in the curve is simply a reflection of the initial condition that much of the carbon was atomic, whereas the second peak is a consequence of freeze-out.

$\mathrm{H}_{2} \mathrm{CO}$ is a hydrocarbon predicted to have a fairly constant fractional abundance as elemental oxygen and carbon freeze-out occurs. Observational studies of this molecule toward the star-containing dense core B335 have revealed a $\mathrm{H}_{2} \mathrm{CO}$ line profile that is consistent with collapse. ${ }^{16}$

Collapse signatures have also been detected toward B335 in CS line profiles. ${ }^{13}$ As mentioned above, sulfur and metals seem to freeze out in ways that differ markedly from those associated with carbon, nitrogen, and oxygen. To interpret the CS profiles, we need to know whether further elemental sulfur freeze-out occurs as the depletion of less massive elements becomes substantial.

The line profiles of many species important for the diagnosis of collapse depend on the ratio of the dynamic time scale to the time scales for the freeze-out of various species. All of the freeze-out time scales remain to be inferred from experimental and theoretical studies.

\section{Cosmic Dust and the Gas/Surface Interaction}

3.1. The Nature of the Dust Surface The evidence ${ }^{17}$ for the widespread existence of interstellar dust leads to the conclusion that about $1 \%$ of the mass of the interstellar medium of the Galaxy is in dust, that dust grains range in size from a few nanometers to at least a few tenths of a micrometer, and that their size distribution decreases steeply with sizea, perhaps as $a^{-3.5}$. The dust composition in diffuse clouds certainly includes silicates, hydrogenated amorphous carbon, and possibly graphite. In sufficiently opaque regions, through which the optical depth to visual radiation is more than about $2, \mathrm{H}_{2} \mathrm{O}$ ice mantles are deposited on the refractory dust. The ice is detected by absorption in the ice band near $3 \mu \mathrm{m}$ and elsewhere. ${ }^{6}$ More heavily extinguished regions exhibit absorption by solid CO, near $4.7 \mu \mathrm{m}$. Other species are also present in the ice (e.g., $\left.\mathrm{CH}_{3} \mathrm{OH}\right) .{ }^{18}$
The dust grains are almost certainly porous, open structures. Such a morphology is found for some interplanetary dust particles. The void component may be significant and the surface area larger than the equivalent for a sphere of the same mass. Variations in the optical properties of dust from one line of sight to another can be accounted for-but only in part-by changes in the size distribution of the grains. It appears that changes in the optical properties of the dust grains are also required. ${ }^{19}$ These changes may be achieved by photoprocessing of carbonaceous materials. 20

3.2. Sticking at Dust Surfaces. The fundamental process in the gas/surface interaction is sticking. What is the probability that a gas atom or molecule becomes bound on the surface? The assumption generally made by astrophysicists is that the probability is of order unity. Theoretical studies show that it is sensitive to the interaction potential, to the energy exchange mechanisms available, and to the temperatures of gas and surface. Early work discussed adsorption on crystalline materials. ${ }^{21}$ Theoretical studies on amorphous materials have concentrated on the interaction of $\mathrm{H}$ and $\mathrm{H}_{2}$ on amorphous ice. Initial studies involving amorphous carbon surfaces have been completed. 22

Several studies were made through classical molecular dynamics (MD) simulations of the interaction of $\mathrm{H}$-atoms with amorphous ice.23,24 These studies generally imply that the sticking probability is of order unity under conditions relevant to the cool interstellar medium. In the most detailed study 24 the MD-stimulated amorphous ice matches well the experimental data on low-temperature ice, and the sticking probability on ice at $10 \mathrm{~K}$ is $1.0,0.98$, and 0.53 at temperatures of 10,100 , and $350 \mathrm{~K}$, respectively. The adsorbed $\mathrm{H}$-atom diffuses over the surface by thermal hopping until it is trapped at a site of strong binding. $\mathrm{No} \mathrm{H}$-atom ejection was observed in these simulations. An experiment 25 to determine the sticking of warm $\mathrm{H}$-atoms on cold dirty ice gave a result one order of magnitude smaller.

There is little work reported in the literature on the sticking of heavier species on relevant amorphous surfaces, although the astrophysical consequences of sticking may be profound. It is generally assumed in astrochemical models that saturated neutral molecules, such as $\mathrm{H}_{2} \mathrm{O}$, $\mathrm{NH}_{3}, \mathrm{CH}_{4}, \mathrm{H}_{2} \mathrm{CO}, \mathrm{CH}_{3} \mathrm{OH}, \mathrm{HCN}$, etc., stick with probability equal to unity. The interaction of neutral atoms is often assumed to lead to hydrides, but it is unclear whether the products are released to the gas phase or retained on the dust grain (d):

$$
\mathrm{O} \stackrel{\mathrm{d}}{\rightarrow} \mathrm{O}-\mathrm{d} \stackrel{\mathrm{H}}{\rightarrow} \mathrm{H}_{2} \mathrm{O}-\mathrm{d} \rightarrow \mathrm{H}_{2} \mathrm{O} \uparrow+\mathrm{d}
$$

The detection of interstellar ice for clouds of sufficient optical opacity suggests that much of the $\mathrm{H}_{2} \mathrm{O}$ must be retained in those circumstances, ${ }^{26}$ although this does not happen in diffuse clouds. However, it is unclear at present whether release occurs as the saturated hydride (e.g., $\mathrm{H}_{2} \mathrm{O}$, $\left.\mathrm{NH}_{3}\right)$ or as an unsaturated radical $\left(\mathrm{OH}, \mathrm{NH}, \mathrm{NH}_{2}\right)$. The case of sulfur atoms is particularly interesting, and as 
described in section 2.2, may have significant consequences in star-forming regions.

Beyond the assumptions described above for atoms and molecules, there is no consensus among astrophysicists on how to treat the interaction of low-temperature atomic and molecular ions with dust grains. Here we list the various channels that may be open. For atomic ions, there are circumstances when they may interact with grains of positive, neutral, or negative charge:

$$
\begin{aligned}
X^{+}+d^{-} \rightarrow & X \uparrow+d \\
& X-d \\
& X^{+}+d^{-} \\
X^{+}+d^{0} \rightarrow & X+d^{+} \\
& X^{+}-d \\
& X-d^{+} \\
& X^{+}+d^{\circ} \\
X^{+}+d^{+} \rightarrow & X^{+}-d^{+} \\
& X-d^{2+} \\
& X^{+}+d^{+}
\end{aligned}
$$

If, in eq 6 , the ion recombination occurs at long range, then the atom-surface interaction is essentially as discussed above and the sticking probability could be assessed on that basis. On the other hand, if the recombination occurs at short range, the energy release may inhibit sticking. In eq 7, the ion charge may promote the atom-surface interaction and enhance sticking. Interaction eq 8 is certainly less favorable, but there may be possible attractive avenues of approach. ${ }^{27}$

The interaction of molecular ions with dust grains may be important in dark clouds where dust grains are mostly negative and where the fractional ionization is very low $\left(\sim 10^{-8}\right)$. Various channels are indicated for $\mathrm{HCO}^{+}$, as an example:

$$
\begin{aligned}
\mathrm{HCO}^{+}+\mathrm{d}^{-} \rightarrow & \mathrm{HCO} \uparrow+\mathrm{d} \\
& \mathrm{HCO}-\mathrm{d} \\
& \mathrm{CO} \uparrow+\mathrm{d}-\mathrm{H} \\
& \mathrm{CO} \uparrow+\mathrm{H} \uparrow+\mathrm{d} \\
& \mathrm{CO}-\mathrm{d}+\mathrm{H} \uparrow
\end{aligned}
$$

However, nothing is known (by astrophysicists) about the probabilities of such interactions.

\section{Formation of $\mathrm{H}_{2}$ on Dust Grains}

An estimate of the rate of formation of $\mathrm{H}_{2}$ in the interstellar medium can be made by assuming steady state and calculating the rate of destruction. Molecular hydrogen is destroyed in the diffuse interstellar medium through line absorption at wavelengths near $100 \mathrm{~nm}$ from the ground state $X$ to various rovibrational states of the excited electronic levels B and C. ${ }^{28}$ The excited molecule relaxes into a distribution of rovibrational levels of state $\mathrm{X}$,

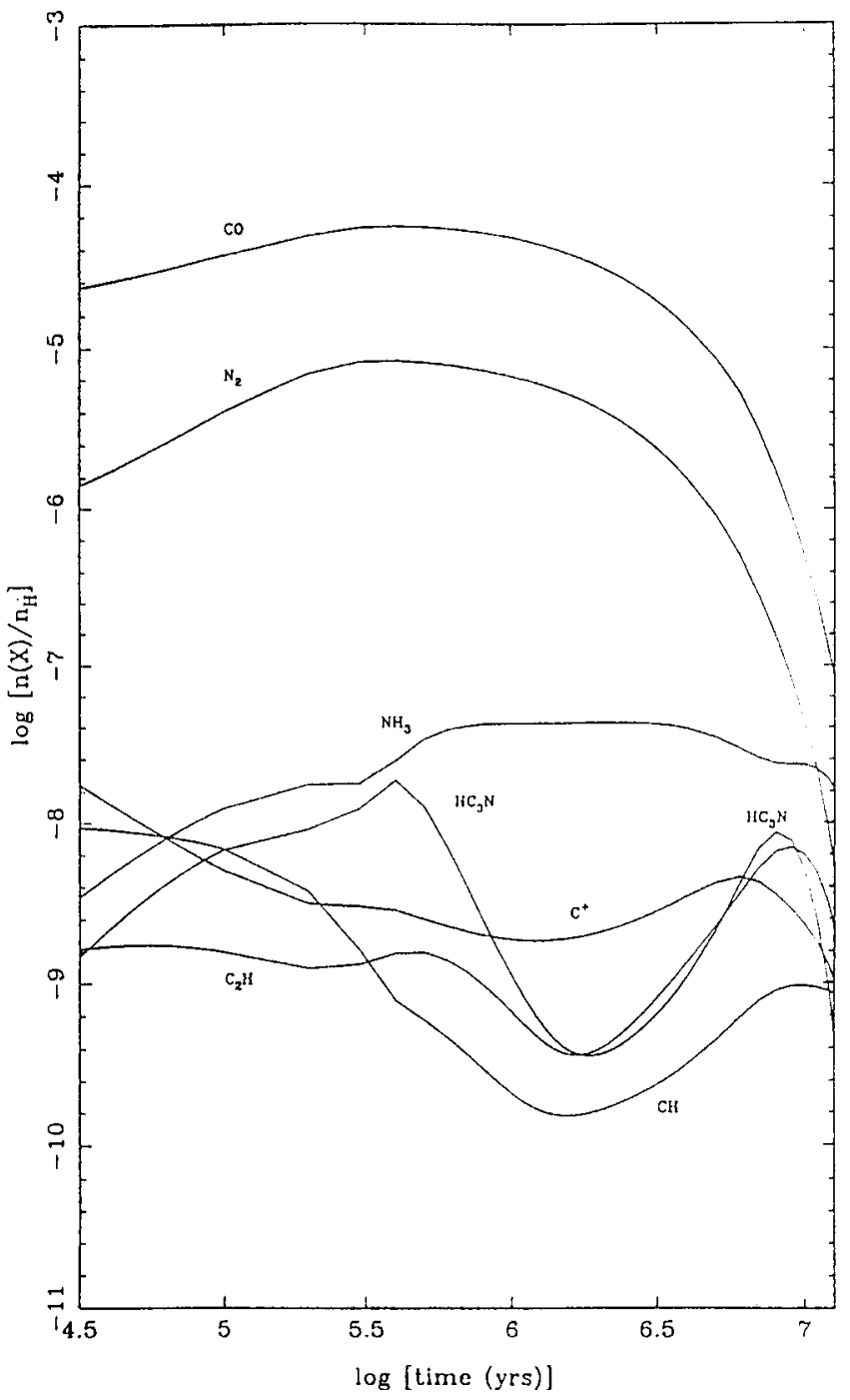

FIGURE 3. The computed time-evolution of functional abundances of various species in static dense core. Initially, all elements other than hydrogen are atomic. Molecules formed in the chemistry are lost by freeze-out onto dust on a time scale assumed here to be about 3 million years; $\mathrm{CO}$ and $\mathrm{N}_{2}$ illustrate this behavior. Other species show a late-time peak in abundance (e.g., cyanopolyyne, $\mathrm{HC}_{3} \mathrm{~N}$; see section 2.3). The early-time peak in $\mathrm{HC}_{3} \mathrm{~N}$ reflects the assumed initial atomic condition of the gas. Reprinted with permission from ref 15. Copyright 1997 M arston Book Services.

including the vibrational continuum. These continuum states are dissociative. Because the destruction process is initiated by a line excitation, $\mathrm{H}_{2}$ may self-shield. In the optically thin case, however, the photodissociation rate is well determined, and together with the observed abundances of $\mathrm{H}$ and $\mathrm{H}_{2}$, the formation rate ${ }^{29}$ in these regions may be written as about $3 \times 10^{-17} \mathrm{n}_{\mathrm{H}} \mathrm{n}(\mathrm{H}) \mathrm{cm}^{-3}$ $\mathrm{s}^{-1}$, where $n_{H}$ and $n(H)$ are the number densities of all hydrogen nuclei and of free $\mathrm{H}$-atoms, respectively.

This observational requirement for the general interstellar medium cannot be met by any known gas phase reactions. ${ }^{32}$ Surface processes, however can do so if they are of high efficiency; i.e., if nearly all $\mathrm{H}$-atoms arriving at grain surfaces are incorporated into $\mathrm{H}_{2}$ molecules and ejeccted from the surface. 
Energy deposition during $\mathrm{H}_{2}$ formation in postshock regions and in gas exposed to $\mathrm{X}$-rays is of great importance for the thermal structures and emissivities of a variety of astrophysical sources. For instance, the release of energy in the production of $\mathrm{H}_{2}$ in $\mathrm{H}_{2} \mathrm{O}$ masers in shocked gas near recently born high-mass stars has been proposed as the mechanism that establishes thermal conditions required for collisional pumping of the masers. ${ }^{30}$ Also, excited rovibrational levels of $\mathrm{H}_{2}$ in gas in molecular tori irradiated by nearby active galactic nuclei, probably powered by accretion onto supermassive blackholes, may be populated primarily as a consequence of $\mathrm{H}_{2}$ formation on grains; ${ }^{31}$ the infrared radiation emitted in the decays of such levels is important in the diagnosis of these exotic regions.

There are two generic mechanisms: ${ }^{32}$ in the first, "standard" model, an $\mathrm{H}$-atom arrives at a surface, is adsorbed but mobile, locates another $\mathrm{H}$-atom bound at a special site, the reaction to form $\mathrm{H}_{2}$ occurs, and the energy released ejects an excited $\mathrm{H}_{2}$ molecule from the surface. This process is called the Langmuir-Hinshelwood $(\mathrm{L}-\mathrm{H})$ mechanism. In a second, or "prompt" reaction model, 33 the incident $\mathrm{H}$-atom is not accommodated on the surface but interacts directly (promptly) with a physisorbed or chemisorbed $\mathrm{H}$-atom; it does not move over the surface. This process is called the Eley-Rideal $(E-R)$ mechanism. There are differences in the two types of processes that would have astrophysical consequences. The $\mathrm{L}-\mathrm{H}$ process is sensitive to the nature of the surface, whereas the $E-R$ process is not. The near uniformity on many lines of sight of the $\mathrm{H}_{2}$ formation rate implied by observational studies may therefore favor the $\mathrm{E}-\mathrm{R}$ mechanism. There are also different temperature dependences for each process which may be important when $\mathrm{H}_{2}$ formation is considered in a range of astrophysical circumstances.

Recent advances in both experimental and computational techniques have made it possible to investigate for the first time the $\mathrm{H}_{2}$ formation process under circumstances that are reasonable approximations to interstellar conditions. Studies of reactions on silicates suggest that the $\mathrm{H}_{2}$ formation rate is significantly smaller than that required by the observations. ${ }^{34,35}$ The discrepancy may possibly be removed if the grains have a larger surface area than assumed, i.e., are porous. A theoretical study of $\mathrm{H}_{2}$ formation on carbon grains shows that a variety of pathways operate under a broad range of conditions. ${ }^{36} \mathrm{~A}$ theoretical study of $\mathrm{H}_{2}$ formation on amorphous ice, ${ }^{37}$ incorporating the results of the MD description of the ice and of $\mathrm{H}$-atom interactions with it, leads to the following conclusions. The $\mathrm{H}$-atom sticking probability is generally high. Several reaction types were observed to occur, including the $\mathrm{L}-\mathrm{H}$ and $\mathrm{E}-\mathrm{R}$ mechanisms. In some cases, the molecule is not formed; however, when part of the excess energy released by formation can be absorbed by the ice, then the reaction occurred and the $\mathrm{H}_{2}$ molecule was formed. The reaction probabilities found in these simulations were very close to unity at 10 and $70 \mathrm{~K}$. Most of the ejected $\mathrm{H}_{2}$ molecules were found to be in states of high rovibrational excitation, in this simulation. ${ }^{38}$ Signifi- cant vibrational excitation of $\mathrm{H}_{2}$ is also predicted by theoretical studies of $\mathrm{H}_{2}$ formation on graphite. ${ }^{22}$

\section{Desorption Processes}

Thermal desorption of ice mantles on dust evidently occurs in so-called hot cores (as distinct from cold dense cores which may evolve to form stars). Their chemistry is consistent with the evaporation and processing of ices that have been deposited in an early phase. The evaporation is, presumably, thermal and is caused by the presence of a nearby newly formed massive star (cf. section 2.1).

Nonthermal desorption may also occur, and could be significant in offsetting the effects of freeze-out. As Table 1 indicates, the freeze-out time scale may be very short, so short that the chemical development of the clump may be inhibited. There is some evidence from chemical modeling that an "effective" freeze-out time longer than implied by Table 1 is required. ${ }^{39,40}$

Possible energy sources for desorption from ices deep in molecular clouds include the following: 42 ionization by cosmic rays, photoabsorption of radiation generated by cosmic rays, and chemical energy. All of these sources may play a role.

In principle, any exothermic reaction occurring at the surface of a dust grain must cause local heating around the site of the reaction. M olecules adsorbed in the vicinity may be evaporated as a result of this energy increment. 41 It appears that the formation of $\mathrm{H}_{2}$ is in many circumstances the most frequently occurring reaction; energy of about $4.5 \mathrm{eV}$ must be distributed between the reaction site, and translational and internal energy in the molecule. If only three additional molecules per $100 \mathrm{H}_{2}$ forming events are desorbed, then this process is faster than either of the competing mechanisms. ${ }^{42}$ There is some suggestion from modeling studies that the process may be even more efficient, and that these would be important consequences for both solid and gas phase chemistry. ${ }^{43}$ In an MD simulation of $\mathrm{H}_{2}$ formation, ${ }^{38}$ about $5 \%$ of the bond energy is found to be absorbed locally in the surface. This would be enough to drive the nonthermal mechanism of desorption described here.

However, no direct test of these ideas for material of astrophysical interest has yet been made in the laboratory, although the energy deposition into an amorphous ice mantle has been computed in the studies of $\mathrm{H}_{2}$ formation. The almost complete lack of knowledge about the effectiveness of chemical desorption is probably the single most important barrier to our understanding of the physics and chemistry of star formation.

\section{Conclusions}

We have described the main physical processes in the evolution of interstellar gas from a more diffuse clump state to a dense star-forming core. The chemistry plays a crucial controlling role in this evolution by providing cooling molecules (which limit the thermal support) and by determining the level of ionization (and therefore the extent of magnetic and turbulent support). Although our 
knowledge of the gas phase processes during this evolution is not complete, nevertheless, the processes are in general reasonably well understood. By contrast, the gas/ dust interaction is poorly understood, yet-as argued here-this interaction is important for heterogeneous catalysis, and for formation of ices and loss of molecules to the gas phase with consequent effects on cooling and fractional ionization. The role of chemical energy in driving a continuous desorption of molecules from ices may be exceptionally important to these processes, but our ignorance of the gas/dust interactions, in terms of sticking probability, mobility of adsorbed species, and the surface reaction process, is almost total. However, recent advances in both experimental and computational techniques have now made these processes accessible to detailed study. We may, therefore, expect rapid developments in the study of gas/surface interactions of relevance to astronomy, as was stimulated for gas phase chemistry by the demands of astrochemistry.

\section{References}

(1) van Dishoeck, E. F. The chemistry of diffuse and dark interstellar clouds. In The Molecular Astrophysics of Stars and Galaxies; Hartquist, T. W., Williams, D. A., Eds.; Oxford University Press: Oxford, 1998, in press.

(2) Herbst, E. Chemistry of the interstellar medium. Ann. Rev. Phys. Chem. 1995, 46, 27-53.

(3) Hartquist, T. W.; Caselli, P.; Rawlings, J. M. C.; Ruffle, D. P.; Williams, D. A. The chemistry of star forming regions. In The Molecular Astrophysi cs of Stars and Galaxies; Hartquist, T. W., Williams, D. A., Eds.; Oxford University Press: Oxford, 1998.

(4) Williams, J. P.; Blitz, L.; Stark, A. A. The density structure in the Rosette Molecular Cloud: signposts of evolution. A. A. Astrophys. J. 1995, 451, 252-274.

(5) Goldsmith, P. F.; Langer, W. D.; Wilson, R. Molecular outflows, gas density distribution, and the effects of star formation in the dark cloud Barnard 5. Astrophys. J. 1986, 303, L11-L15.

(6) Whittet, D. C. B. Dust in the Galactic Environment, IOP Publishing: Bristol, 1992.

(7) Mathis, J. S. Interstellar dust and extinction. Ann. Rev. Astron. Astrophys. 1990, 28, 37-70.

(8) Meyer, D. M.; Roth, K. C. Discovery of interstellar NH. Astrophys. J. Lett. 1991, 376, L49-L52.

(9) Crawford, I. A.; Williams, D. A. Detection of interstellar NH towards $\xi$ Ophiuchi by means of ultrahigh-resolution spectroscopy. Mon. Not. R. Astron. Soc. 1997, 291, L53-L56.

(10) Wagenblast, R.; Williams, D. A.; Millar, T. J.; Nejad, L. A. M. On the origin of $\mathrm{NH}$ in diffuse interstellar clouds. Mon. Not. R. Astron. Soc. 1993, 260, 420424.

(11) Millar, T. J. The chemistry of hot molecular cores. In Dust and Chemistry in Astronomy; Millar, T. J., Williams, D. A., Eds; Institute of Physics Publishing: Bristol, 1993; pp 249-269.

(12) Hartquist, T. W.; Williams, D. A., Eds.; In The Chemcially Controlled Cosmos; Cambridge University Press: Cambridge, 1995.

(13) Ruffle, D. P.; Hartquist, T. W.; Rawlings, J. M. C.; Williams, D. A. Ionization structure and a critical visual extinction for turbulent supported clumps. Astron. Astrophys. 1998, 334, 678-684.

(14) Myers, P.; Benson, P. J. Dense cores in dark clouds. II $-\mathrm{NH}_{3}$ observations and star formation. Astrophys. J. 1983, 266, 309-320.

(15) Ruffle, D. P.; Hartquist, T. W.; Taylor, S. D.; Williams, D. A. Cyanopolyynes as indicators of late-time chemistry and depletion in star-forming regions. Mon. Not. R. Astron. Soc. 1997, 291, 235-240.

(16) Zhou, S. In search of evidence for protostellar collapse: a systematic study of line formation in low-mass dense cores. Astrophys. J. 1992, 394, 204216.

(17) Williams, D. A.; Taylor, S. D. The chemical role of cosmic dust. Q. J. R. Astron. Soc. 1996, 37, 565-592.

(18) Whittet, D. C. B.; et al. An ISO SWS view of interstellar ices: first results. Astron. Astrophys. 1996, 315, L357-L360.

(19) Cecchi-Pestellini, C.; Williams, D. A. Evolving interstellar extinction. Mon. Not. R. Astron. Soc. 1998, 296, 414-418.

(20) Duley, W. W. Carbonaceous grains. In Dust and Chemistry in Astronomy; Millar, T. J., Williams, D. A., Eds.; Institute of Physics Publishing: Bristol, 1993, pp 71-101.

(21) Leitch-Devlin, M. A.; Williams, D. A. Sticking coefficients for atoms and molecules at the surfaces of interstellar dust grains. Mon. Not. R. Astron. Soc. 1985, 213, 295-306.

(22) Farebrother, A.; Clary, D. C.; Fisher, A. Eley-Rideal reaction of hydrogen atoms with hydrogen adsorbed on graphite at very low temperatures. 1998. Manuscript in preparation.

(23) Buch, V.; Zhang, Q. Sticking probabilities of $\mathrm{H}$ and $\mathrm{D}$ atoms on amorphous ice: a computational study. Astrophys. J. 1991, 379, 647-652.

(24) Masuda, K.; Takahashi, J.; Mukai, T. Sticking probability and mobility of a hydrogen atom on icy mantle of dust grains. Astron. Astrophys. 1998, 330, 773-781.

(25) Mattera, L. Interaction of $\mathrm{H}, \mathrm{H}_{2}$, and $\mathrm{D}_{2}$ beams with solid surfaces at low temperatures. Ph.D. Thesis, University of Waterloo, Canada, 1978.

(26) Jones, A. P.; Williams, D. A. The $3 \mu \mathrm{m}$ ice band in Taurus: implications for interstellar chemistry. Mon. Not. R. Astron. Soc. 1984, 209, 955-960.

(27) Jones, A. P.; Williams, D. A. Electric charge on ionic grains. Mon. Not. R. Astron. Soc. 1986, 219, 441447.

(28) Stecher, T. P.; Williams, D. A. Photodestruction of hydrogen molecules in HI regions. Astrophys. J. Lett. 1967, 149, L29-L30.

(29) Jura, M. Interstellar clouds containing optically thin $\mathrm{H}_{2}$. Astrophys. J. 1975, 197, 575-580.

(30) Elitzur, M.; Hollenbach, D. J.; McKee, C. F. $\mathrm{H}_{2} \mathrm{O}$ masses in star forming regions, Astrophys. J. 1989, 346, 983-990.

(31) Lepp, S.; Tiné, S. X-ray dominated regions. In The Molecular Astrophysics of Stars and Galaxies; Hartquist, T. W., Williams, D. A., Eds.; Oxford University Press, 1998, in press.

(32) Duley, W. W.; Williams, D. A. Interstellar Chemistry; Academic Press, London, 1984.

(33) Duley, W. W. The formation of $\mathrm{H}_{2}$ by $\mathrm{H}$-atom reaction with grain surfaces. Mon. Not. R. Astron. Soc. 1996, 279, 591-594.

(34) Pirronello, V.; Liu, C.; Shen, L.; Vidali, G. Laboratory synthesis of molecular hydrogen on surfaces of astrophysical interest. Astrophys. J. Lett. 1997, 475, L69-L72. 
(35) Pirronello, V.; Biham, O.; Liu, C.; Shen, L.; Vidali, G. Efficiency of molecular hydrogen formation on silicates. Astrophys. J. Lett. 1997, 483, L131.

(36) Levinson, A.; Chernoff, D. F.; Salpeter, E. E. Molecule formation in and on grains. I: Physical Regimes. Astrophys. J. 1998, in press.

(37) Takahashi, J.; Masuda, K.; Nagoaka, M. Formation mechanism of molecular hydrogen on icy mantles of interstellar dust. Mon. Not. R. Astron. Soc., in press.

(38) Takahashi, J.; Masuka, K.; Nagaoka, M. Product energy distribution of molecular hydrogen formed on icy mantles of interstellar dust. Astrophys. J., in press.

(39) Taylor, S. D.; Morata, O.; Williams, D. A. The distribution of $\mathrm{CS}$ and $\mathrm{NH}_{3}$ in star-forming regions. Astron. Astrophys. 1996, 313, 269-276.
(40) Howe, D.; Taylor, S. D.; Williams, D. A. Chemical interpretation of the sequential star formation hypothesis. Mon. Not. R. Astron. Soc. 1996, 279, 143-151.

(41) Allen, M.; Robinson, G. W. Formation of molecules on small interstellar grains. Astrophys. J. 1975, 195, 81-90.

(42) Willacy, K.; Williams, D. A. Desorption processes in molecular clouds: quasi-steady state chemistry. Mon. Not. R. Astron. Soc. 1993, 260, 635-642.

(43) Willacy, K.; Williams, D. A. Duley, W. W. Desorption of grain mantles by $\mathrm{H}_{2}$ formation. Mon. Not. R. Astron. Soc. 1994, 267, 949-956.

AR9701140 